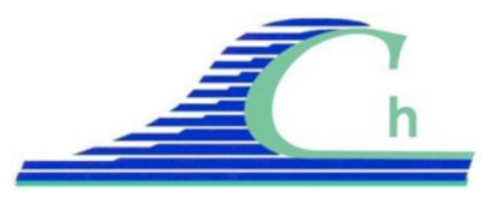

XII ${ }^{\text {èmes }}$ Journées Nationales Génie Côtier - Génie Civil

Cherbourg, 12-14 juin 2012

DOI:10.5150/jngcgc.2012.077-E_ @ Editions Paralia CFL

disponible en ligne - http://www.paralia.fr - available online

\title{
Traitement des remblais des terre-pleins au nouveau port Tanger Med II
}

\author{
Taoufik EL OUAHABI ${ }^{1}$
}

1. Société Tanger Med Engineering (TME), Zone franche Ksar Al majaz, Tanger Med, Maroc.

t.elouahabi@tme.ma

\section{Résumé :}

Dans cette contribution, on va aborder les différents matériaux constituant le terre-plein du port Tanger Med II et on va détailler particulièrement la méthodologie adoptée pour traiter ces matériaux afin de respecter les exigences contractuelles en matière de tassement et de portance. Il s'agit dans le premier cas d'un remblai en stérile de carrière 0/300 mm dont une étude a été menée pour vérifier premièrement sa vibrocompactabilité et deuxièmement pour arrêter les critères d'acceptation en conformité avec le contrat. Les résultats de cette étude seront rappelés brièvement.

Par la suite, il faut choisir le vibreur adéquat qui permet d'atteindre d'une manière optimale les objectifs. Le choix se base sur des critères techniques comme l'amplitude et la fréquence de vibration.

Pour la partie réalisation et avant d'entamer les travaux en zone définitive, il fallait d'abord vérifier les résultats de l'étude technique en grandeur nature moyennant des planches d'essais en utilisant le matériel qui sera destiné au traitement final. Durant cette phase, le couple maillage / durée de vibration sera arrêté.

La réussite des étapes précédentes a permis de passer à la phase exécution en toute sérénité. Les travaux ont duré 2 mois et demi et les résultats était sans surprise conformes aux attentes.

Pour le remblai hydraulique provenant du dragage du fond marin. La technique est la même mais la méthodologie de la réalisation et de la réception est complètement différente.

\section{Mots clés :}

Terre-plein - Remblais hydrauliques - Vibrocompactabilité - Densité relative Résistance à la pénétration statique - Contrôle qualité

\section{Introduction}

Le terre-plein du nouveau port Tanger Med II, spécialisé en conteneurs, est d'une superficie de 76 ha en première phase et de 150 ha en seconde phase. La réalisation de ces travaux est à la charge d'un consortium d'Entreprises (BOUYGUES, BYMARO, SAIPEM, BESIX et SOMAGEC). Dans cet article, l'expérience vécue au chantier TM2 est rapportée en matière de la méthodologie pour la réalisation de ces terre-pleins en 
conformité avec les exigences contractuelles. Les contraintes et les solutions apportées seront exposées en s'attardant sur un volet majeur: le vibrocompactage. Le contrôle qualité assuré par les équipes Tanger Med Engineering (TME) durant toutes les étapes de la réalisation est également abordé.

La réalisation des terre-pleins du futur port Tanger Med II était prévue à partir de la fin de l'an 2012 moyennant du matériau du remblai hydraulique en provenance d'un gisement pas loin du site. Cependant pour obtenir les terrains nécessaires aux installations d'un grand chantier, le consortium d'entreprises a fait le choix de les gagner sur la mer. Il s’agit donc de réaliser des digues d'enclôture et de remplir le bassin par remblai hydraulique. Cette opération prévue et sensée démarrer dès l'ouverture de chantier a été vite abandonnée à cause des délais nécessaires pour l'obtention des autorisations de l'ouverture et de l'exploitation des gisements sousmarins. C'est pour cette raison que le consortium a constitué une partie de ces terrepleins en remblais sableux disponibles et le reste en remblai (deux millions de tonnes) avec un matériau composé de stérile de carrière $0 / 300 \mathrm{~mm}$ en provenance d'une carrière à environ $10 \mathrm{~km}$ du chantier.

Les caractéristiques granulométriques du matériau 0/300 mm ont montré leur capacité à la vibrocompactabilité. Cette technique très répandue a pour objectif de diminuer l'indice de vide et par conséquent d'augmenter la densité relative des matériaux granulaires constituant le remblai en réarrangeant les grains du sol par vibration. Une étude a été également menée pour arrêter l'équivalent du critère d'acceptation (CPT >10 $\mathrm{MPa}$ ) applicable au type de matériau mis en place. Il s'agit d'une densité relative de l'ordre de $77 \%$ et un tassement de $6,5 \%$ de la hauteur traitée.

Une fois l'étude technique achevée, il aurait fallu choisir le matériel adapté pour atteindre les objectifs fixés. Le choix s'est alors porté sur le vibreur V48 pour ses performances et son coefficient de pénétration important. L'étape suivante a consisté à la détermination du couple maillage et du temps de vibrocompactage qui se fait à l'aide de planches d'essais. L'objectif est d'adopter un couple optimal tout en fixant une limite d'ampérage comme critère d'arrêt.

Le remblai hydraulique sableux a fait également l'objet du même traitement mais avec une méthodologie et des critères de réception complètement différents.

\section{Recueil contractuel et bibliographique}

\subsection{Critères de réception des terre-pleins}

Plusieurs critères de réception sont déterminés par le contrat de travaux de TM2, à savoir :

- Une valeur minimale de résistance à la pénétration de $10 \mathrm{MPa}$ sur toute la hauteur du remblai à partir de $2 \mathrm{~m}$ de profondeur. 


\section{XII ${ }^{\text {èmes }}$ Journées Nationales Génie Côtier - Génie Civil \\ Cherbourg, 12-14 juin 2012}

- Une valeur de EV2 > $100 \mathrm{MPa}$ (essais à la plaque) et un rapport (EV2/EV1)<2 sont exigés pour les $50 \mathrm{~cm}$ supérieurs du remblai supérieur.

- En plus, une note technique doit être élaborée et validée pour vérifier les tassements à court et à long termes. Les tassements admissibles maximaux au niveau de la surface réceptionnée doivent être inférieurs à 75 mm en un an et à 150 mm en 20 ans.

\subsection{Rappel de l'apport du vibrocompactage}

Le vibrocompactage est le procédé qui permet d'exécuter le compactage en profondeur du sol en place, à l'aide d'une aiguille vibrante avec ajout ou non de matériau pendant la phase de traitement. Les grains du matériau lâche sont réarrangés sous l'effet simultané des vibrations et de la saturation en induisant un cisaillement et/ou une liquéfaction localisée lorsque le matériau granulaire est fin.

Ce procédé a été inventé en 1934 en Allemagne par STEUERMAN ET DEGAN (DEBATS, 2003).

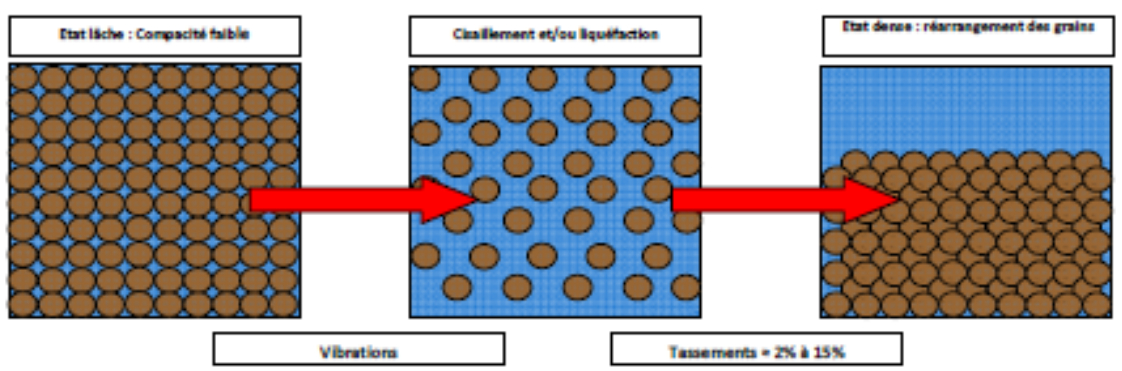

Figure 1. Evolution du matériau lors du vibrocompactage.

Le vibrocompactage se fait en trois étapes qui sont successives :

i. La pénétration (ou fonçage) de l'aiguille vibrante à la profondeur exigée sous l'effet des vibrations et un lançage à l'eau ou à l'air.

ii. L'aiguille vibrante est remontée de la profondeur maximale par passes définies. Le sol en place s'achemine alors vers la pointe de l'aiguille vibrante.

iii.Le compactage est effectué avec un remblaiement depuis la surface avec le sol en place dont la surface subit un affaissement.

Le traitement implique une diminution de l'indice des vides et se traduit donc par une augmentation de densité relative. Cette dernière notion est une des plus importantes dans le domaine de l'amélioration des sols granulaires.

\section{Etude technique}

Une étude technique a été réalisée pour vérifier deux points importants: la vibrocompactabilité du matériau et l’adaptation des critères de réception. 


\subsection{Etude de vibrocompactabilité}

Le choix du type de traitement d'un matériau est fonction de sa nature granulaire. Un sol granulaire est vibrocompactable lorsque celui-ci comprend moins de $10 \%$ de fines (particules dont le diamètre est inférieur à $0.075 \mathrm{~mm}$ ) et un pourcentage d'argile inférieur à 2\%. De plus, un sol dont le coefficient d'uniformité est important (bien gradué) se comporte mieux lors du compactage. Cela s'explique par un meilleur angle de frottement global qui permet la bonne propagation des ondes de cisaillement induites par le vibreur. La figure 2 précise les domaines d'application du vibrocompactage en termes de granulométries.

Des analyses granulométriques ont été réalisées par le laboratoire de chantier sur 18 échantillons des matériaux mis en place suivant la norme NF P 94-051. Ces essais de laboratoire permettent de caractériser la nature des sols en place. La vibrocompactabilité de ces matériaux peut alors être évaluée.

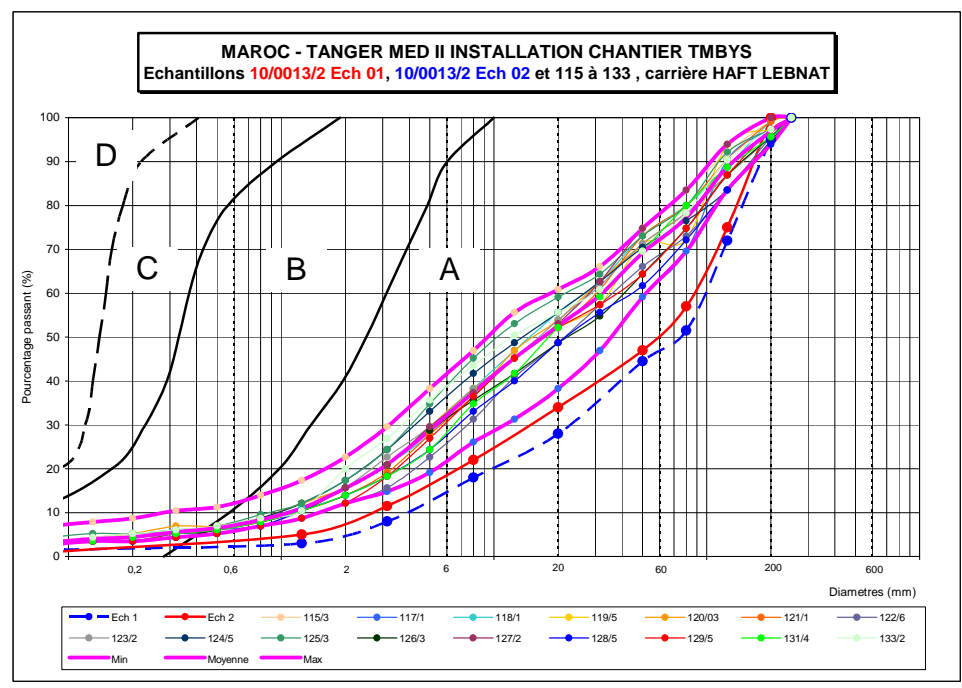

Figure 2: Granulométries et limites de vibrocompactabilité (d'après Vibro Syst Inc).

Le matériau constitutif des terrains en place se situe dans la zone A (voir figure 2) caractérisant des terrains grossiers avec moins de 10\% de particules fines. Par conséquent, les matériaux qui composent ce remblai sont améliorables par vibrocompactage avec des précautions à prendre lors de l'exécution.

\subsection{Adaptation des spécifications de réception}

Compte tenu de la nature des remblais qui sont mis en place $(0-300 \mathrm{~mm})$, le critère de réception exprimé par une résistance en pointe statique ne peut pas s'appliquer. Par conséquent, une approche géotechnique en termes de variation de densité relative liée à la diminution des vides (réduction de l’indice des vides) sera réalisée par le consortium. 


\section{XII ${ }^{\text {èmes }}$ Journées Nationales Génie Côtier - Génie Civil \\ Cherbourg, 12-14 juin 2012}

En effet, une corrélation entre la résistance en pointe, fonction de la contrainte de confinement et donc de la profondeur, et la densité relative a été recherchée. Cela introduit la notion d'évaluation du critère de réception en termes de densité relative, i.e. de réduction d'indice des vides, et par conséquent de tassements immédiats du remblai lors du traitement de vibrocompactage.

En se basant sur la corrélation usuelle de JAMIOLKOWSKI (1985) qui relie la résistance en pointe et contrainte verticale et en représentant le critère contractuel sur le même graphique que la densité relative moyenne correspondante, il a été obtenu que le critère $\mathrm{q}_{\mathrm{c}}=10 \mathrm{MPa}$ est équivalent en moyenne à une densité relative finale théorique de $77 \%$ correspondant à la hauteur moyenne de traitement de $7.50 \mathrm{~m}$ pour l'ensemble du remblai.

En plus, il était nécessaire d'estimer les plages de variation des indices des vides maximum et minimum selon la référence proposée par WINTERKORN \& FANG (1975). Cette variation combinée avec l'estimation de la densité relative du matériau à son état initial (après déversement à l'eau) de l'ordre de $50 \%$ conduit après étude considérant plusieurs hypothèses, à une estimation d'un tassement moyen qui est de l'ordre de 6,5\%.

\section{Choix du matériel et vérification grandeur nature}

\subsection{Le vibreur V48}

Les paramètres caractérisant une vibration (un mouvement oscillatoire) et qui intéressent particulièrement dans le domaine pratique sont : la fréquence des vibrations, l'amplitude maximale du mouvement et la force excentrique mobilisable.

Les caractéristiques du matériel utilisé permettent de déterminer la faisabilité des projets en termes de pénétration, de compactage et de rendement. Le vibreur de type V48 et le vibreur le plus puissant de sa gamme, il a été choisi par le sous-traitant pour ses performances qui sont résumés dans le tableau de la figure 3.

Il y a lieu de noter que le coefficient de pénétration qui définit la capacité d'un vibreur pour pénétrer un sol est calculé à partir d'une formule empirique faisant intervenir l'amplitude, la vitesse et le diamètre de l'appareil :

$$
\frac{10^{-2} \cdot \text { Amplitude }[\mathrm{mm}] \cdot \text { Vitesse }^{2}\left[\mathrm{Tr} \cdot \mathrm{min}^{-1}\right]}{\text { Diametre }^{2}[\mathrm{~mm}]}
$$




\begin{tabular}{|l|c|}
\cline { 2 - 2 } \multicolumn{1}{c|}{} & $\mathrm{V} 48$ \\
\hline Diamètre [mm] & 384 \\
\hline Longueur [m] & 4.08 \\
\hline Masse [kg] & 2700 \\
\hline Vitesse [tr/min] & 1500 \\
\hline Amplitude [mm] & 42 \\
\hline Force excentrique [kN] & 486 \\
\hline Tension [V] & 440 \\
\hline Puissance Moteur [kW] & 175 \\
\hline Fréquence & $50 \mathrm{~Hz}$ \\
\hline Coefficient de pénétration & 6.6 \\
\hline
\end{tabular}

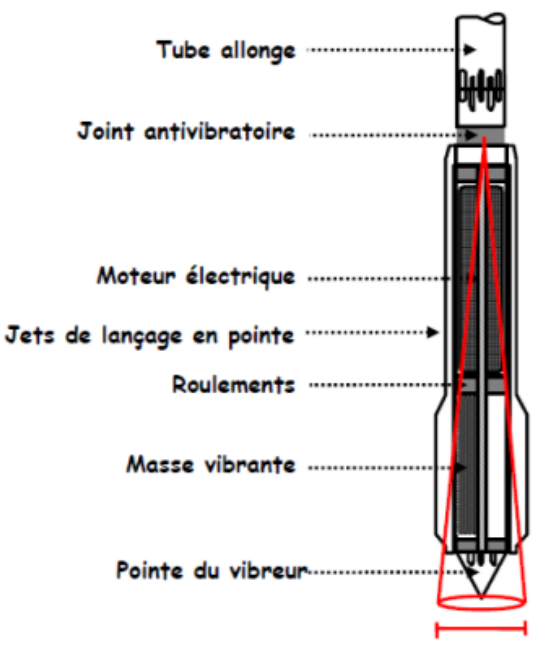

Figure 3. Caractéristiques du vibreur.

\subsection{Planches d'essais}

Ces essais réalisés en début de chantier sont composés de trois mailles de traitement encadrant les mailles principales supposées. Les mailles testées auront pour but de vérifier que le maillage de supposé n'est ni trop lâche ni trop étroit afin de garantir les résultats attendus et de constater les tassements obtenus. Ces tassements résultent du traitement dont l'effet de réarrangement des grains permet d'augmenter la densité relative du matériau.

Sur le plan réel, et pour préserver le vibreur, il faut toujours définir un critère de refus qui est pour notre cas soit l'intensité du moteur électrique qui atteint des valeurs supérieures à 400/450A et/ou la vitesse de pénétration du vibreur qui devient inférieure à $0.5 \mathrm{~m}$ par minute.

De plus, toute l'opération est assistée par un spécialiste qui devra déterminer précisément les rôles joués par l'air et l'eau lors des phases de pénétration et de compactage. Le but final est de déterminer si l'utilisation de l'eau et de l'air sont nécessaires et dans quelles proportions afin d'atteindre les critères spécifiés

\subsection{Déroulement des planches}

Trois mailles ont été testées (3,5 m, 4,0 m et 4,5 m) pour le même temps de vibration qui est de 40 secondes. La vérification des tassements moyens se calcule par deux méthodes. La première est basée sur un levé avant et après traitement, le tassement rapporté à la hauteur du traitement est alors calculé. La seconde consiste à calculer ce coefficient pour chaque point de vibrocompactage.

Les résultats obtenus étaient concluants et la décision a été adoptée pour un maillage de $4 \mathrm{~m}$ et une durée de traitement de $40 \mathrm{~s}$ avec les mêmes critères de refus. Le tassement quant à lui, il a été décidé de le calculer selon la première méthode pour des zones de traitement ne dépassant pas un hectare. 


\section{Réalisation des travaux et contrôle qualité}

La zone de traitement a été découpée en sous zones de 1ha chacune. Un plan de maillage et de zoning a été édité pour être le support unique lors du phasage du traitement. Chaque point est numéroté, un tableau récapitule l'implantation de tous les points.

Lors des travaux, un levé initial est réalisé au droit de chaque point à vibrocompacter, un surveillant observe toutes les séquences de traitement et rapportent les points de contrôle qualité sur une fiche spéciale.

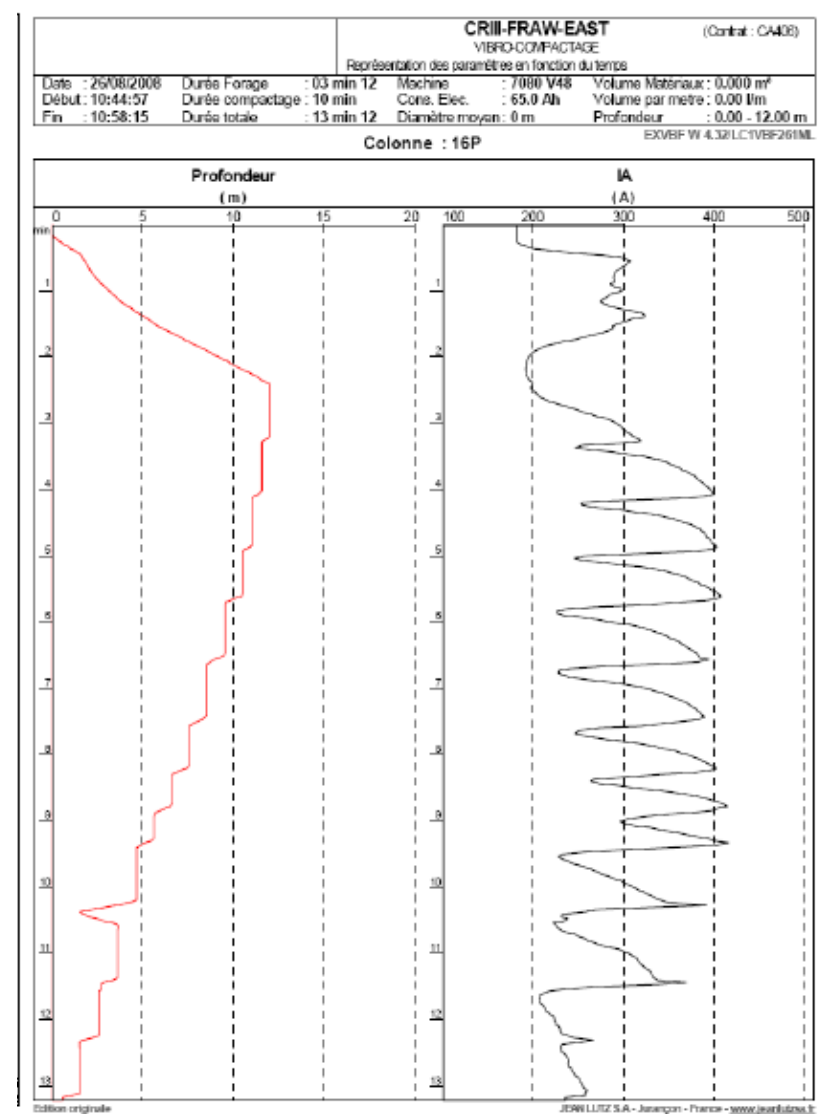

Figure 4. Exemple d'enregistrement en continu des paramètres d'exécution.

Il y a lieu de rappeler que tous les paramètres de traitement sont enregistrés par un enregistreur mis en place au niveau de la grue. Ces enregistrements ont une importance particulière puisqu'ils définissent pour chaque point le temps de pénétration, la profondeur de traitement et l'ampérage atteint. Ces informations sont disponibles sur l'ensemble de la hauteur traitée durant les phases de compactage. Ces enregistrements sont vérifiés un par un pour détecter les anomalies liés au traitement. Un tableau récapitulatif est élaboré en précisant les points validés et les points à refaire. 
La zone est ensuite validée à la base du tassement moyen calculé et qui doit être supérieur à $6,5 \%$.

\section{Cas du remblai hydraulique}

S'agissant d'un remblai hydraulique dont le pourcentage des fines était inférieur à 6\%, le critère d'acceptation (CPT >10 MPa) et la vibrocompactabilité n'étaient pas assujettis à une vérification préalable comme c'était le cas pour le stérile de carrière.

Il fallait uniquement mener des planches d'essais sur le terrain pour définir le couple optimal (maillage, durée de vibrocompactage) ainsi que la méthodologie de traitement. En effet, après plusieurs tentatives défectueuses, les bons résultats obtenus ont été observés en procédant à une humidification préalable de la plateforme au moins 24 heures avant le démarrage du traitement et à l'ajout du sable pendant l'opération. Le maillage requis était de $3,75 \mathrm{~m}$.

A noter que le vibreur remonte à la surface après chaque passe et ce contrairement à la méthodologie du compactage du stérile.

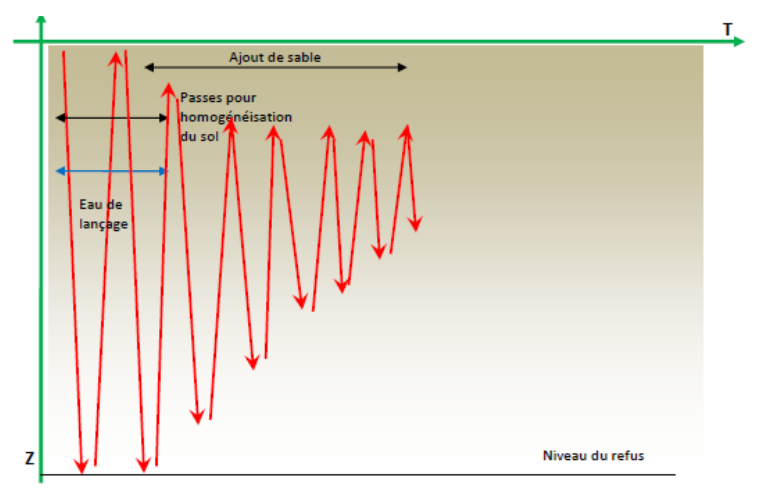

Figure 5. Représentation schématique du vibrocompactage (planche d'essais).

Il faut signaler l'importance du délai de repos avant les essais de contrôle à l'aide d’un pénétromètre statique $\mathrm{CPT}$.

Les résultats des essais $\mathrm{CPT}$ réalisés avant et après vibrocompactage montre l’impact du traitement et le respect du critère contractuel de réception. A gauche un exemple des résultats obtenus. 


\section{XII ${ }^{\text {èmes }}$ Journées Nationales Génie Côtier - Génie Civil \\ Cherbourg, 12-14 juin 2012}

cone resistance (Mpa)

$0 \begin{array}{llllllllll}0 & 2 & 3 & 4 & 5 & 6 & 7 & 8 & 9 & 1011121314151617181\end{array}$

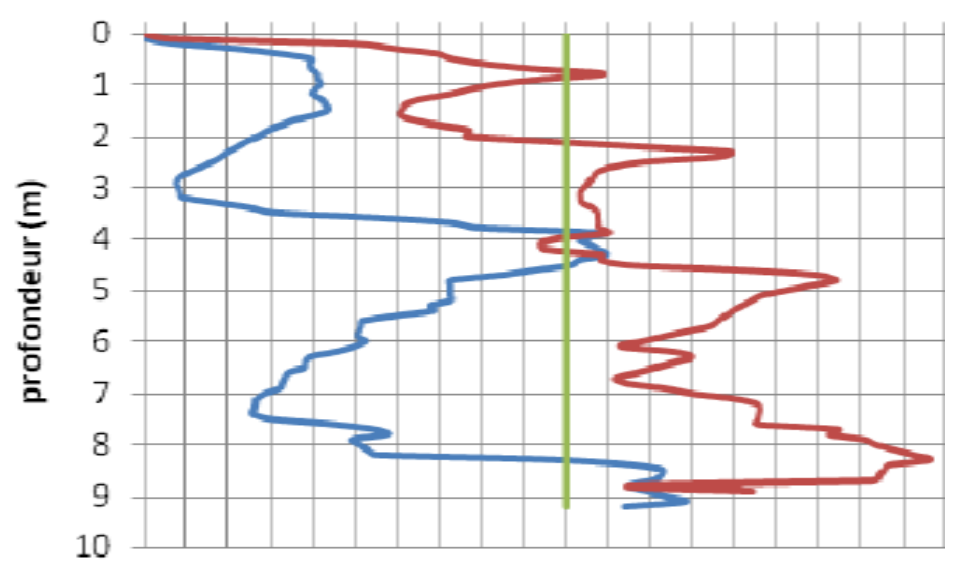

Figure 6. Résultats CPT avant et après traitement.

\section{Conclusion}

La technique du vibrocompactage des matériaux est devenue au fil du temps une technique maîtrisée en termes de consistance et de résultats obtenus. Le traitement des matériaux grossiers (300 mm) s'est avéré selon l'expérience menée au chantier de la réalisation du nouveau port Tanger Med II faisable à conditions de procéder aux vérifications préalables (études de vibrocompactabilité) et d'avoir un matériau extrêmement homogène.

Le choix d'un matériel limite au niveau de ces caractéristiques pose toujours le problème de la fiabilité et de la conformité des résultats finaux d'où l'importance d'une bonne étude au départ du choix du vibrocompcateur. Enfin, pour le cas du projet de TM2, les travaux les plus importants correspondant au traitement d'environ $5000000 \mathrm{~m}^{3}$ (première phase) ne sont pas encore démarrés. Des moyens importants et appropriés seront certainement mobilisés pour respecter les exigences du contrat tout en restant dans les délais impartis.

Il serait également intéressant de suivre au niveau de ce chantier l'expérience du traitement de la partie supérieure du remblai qui se décompacte sous l'effet de la vibration. La méthodologie testée avec réussite consiste en la mise en place d'une couche supérieur de $25 \mathrm{~cm}$ de remblai $0 / 80 \mathrm{~mm}$ de carrière et de compacter l'ensemble par un compactage dynamique réalisé sous l'effet d'un rouleau tracté par engin type Bull adapté. Cette technique a l'avantage de traiter des hauteurs importantes (2 à 2,5 m) au lieu d'une dizaine de centimètres pour le compacteur à rouleau lisse.

\section{Bibliographie consultée}

TM2SA (2009). Contrat EPC études et réalisation des ouvrages de protection et des ouvrages d'accostage du port Tanger Med II. Rapport confidentiel. 
Thème 4 - Ouvrages portuaires et offshore

SOL TM, TMBYS (2010). Note technique d'amélioration des sols par vibrocompactage. Rapport confidentiel.

TMBYS (2010). Procédure de réalisation des travaux de vibrocompactage. Rapport confidentiel.

MENSOY, BSTM, LPEE (2010). Rapport géotechnique des planches d'essais. Rapport confidentiel.

MENSOY, BSTM (2010). Etude et mémoire technique de réalisation des travaux de vibrocompactage. Rapport confidentiel.

WINTERKORN H.F., FANG H.Y. (1975). Foundation Engineering Handbook. Van Nostrand Reinold Ed., 751 p. 